\title{
QUEEN'S
UNIVERSITY
BELFAST
}

\section{Effect of varying dimensions on gadolinium rectangular thin film elements: micromagnetic simulations}

McMullan, M., \& Felton, S. (2018). Effect of varying dimensions on gadolinium rectangular thin film elements: micromagnetic simulations. Journal of Magnetism and Magnetic Materials, 449, 88-93.

https://doi.org/10.1016/j.jmmm.2017.10.009

\section{Published in:}

Journal of Magnetism and Magnetic Materials

\section{Document Version:}

Peer reviewed version

\section{Queen's University Belfast - Research Portal:}

Link to publication record in Queen's University Belfast Research Portal

\section{Publisher rights}

Copyright 2017 Elsevier.

This manuscript is distributed under a Creative Commons Attribution-NonCommercial-NoDerivs License

(https://creativecommons.org/licenses/by-nc-nd/4.0/), which permits distribution and reproduction for non-commercial purposes, provided the author and source are cited.

\section{General rights}

Copyright for the publications made accessible via the Queen's University Belfast Research Portal is retained by the author(s) and / or other copyright owners and it is a condition of accessing these publications that users recognise and abide by the legal requirements associated with these rights.

Take down policy

The Research Portal is Queen's institutional repository that provides access to Queen's research output. Every effort has been made to ensure that content in the Research Portal does not infringe any person's rights, or applicable UK laws. If you discover content in the Research Portal that you believe breaches copyright or violates any law, please contact openaccess@qub.ac.uk. 


\title{
Effect of varying dimensions on gadolinium rectangular thin film elements: micromagnetic simulations
}

\author{
M.F. McMullan \& S. Felton ${ }^{1}$ \\ Centre for Nanostructured Media, School of Mathematics and Physics, Queen's University \\ Belfast, BT7 1NN, U.K
}

\begin{abstract}
Micromagnetic simulations of the ground state magnetization patterns of rectangular gadolinium thin film elements were performed and the effects of aspect ratio, size, and thickness of the island on net long axis moment were investigated. Highly remanent states were found in $15 \mathrm{~nm}$ thick gadolinium islands with high aspect ratios, 8:1 or greater, as well as in smaller islands with aspect ratios down to 5:1. The thickness of the island was also critical for the ability of a gadolinium island to maintain a highly remanent ground state; islands with thickness greater than or equal to $30 \mathrm{~nm}$ showed increased multidomain formation. These results provide information and guidance on what dimensions are suitable for fabrication of single-domain gadolinium nanostructures - a feat not yet achieved in literature. The proposed dimensions most suitable for experimental realisation of a single domain gadolinium element are $500 \mathrm{~nm} \times 50 \mathrm{~nm} \times 15 \mathrm{~nm}$, lying within the range found in this investigation to be energetically favoured single domains.
\end{abstract}

Keywords: Micromagnetism, ferromagnetism, gadolinium, single-domain

\section{Introduction}

Gadolinium $(\mathrm{Gd})$ is a rare earth metal which in hexagonally close packed (hcp) bulk form is notable for its high Curie temperature of $293 \mathrm{~K}$ (for rare earths) and high saturation magnetization value, in part due to a large mag5 netic moment from seven unpaired 4f electrons. [1] In single crystal gadolinium, 
magnetization values of $2.12 \mathrm{MAm}^{-1}$ have been achieved. [1] Gadolinium has applications across disciplines: potential use exist in magnetic refrigeration, [1] as a contrast agent in magnetic resonance imaging, 2] and to aid in the study of effects such as interface anisotropy and interlayer coupling in multilayer thin film devices. [3, 4, 5]

Confining the dimensions of ferromagnetic materials on the nanoscale is well known to have considerable influence on the resultant magnetic properties. 66, 7] The thin film form of gadolinium typically assumes a face centered cubic (fcc) arrangement which is paramagnetic. 8] However, the ferromagnetic hcp form that is found in bulk can be induced through tailored growth conditions. Advances in growth procedures for thin film Gd has resulted in the demonstration of saturation magnetization values akin to those found in single crystals. [9] In the work undertaken by Scheunert et al 9 Gd thin films were grown using DC magnetron sputtering on Si wafers; it was found that a $5 \mathrm{~nm}$ Ta seed layer in combination with an elevated deposition temperature resulted in primarily hcp Gd in the film.

To date the magnetic properties of nanopatterned gadolinium have not been extensively investigated and in literature there have been calls for a better understanding of the magnetic properties of $4 \mathrm{f}-$-elements; 8 this is something this study 25 directly addresses. Previous experimental work on nanopatterned gadolinium includes nanowires fabricated from Gd thin film by focused ion beam with dimensions of $275 \mathrm{~nm} \times 10 \mu \mathrm{m}$ [10] and the magnetotransport properties studied, as well as an unsuccessful attempt to induce single domain states in gadolinium nanobars, 8] where the high aspect ratio structures $\left(1400 \times 70 \times 50 \mathrm{~nm}^{3}\right)$ were used in the hope of inducing a single domain state due to shape anisotropy. 8 The simulations reported in this paper offer an explanation for the failure of that study to produce single domain islands.

With the development of software to predict the magnetic behavior on the nano and micro scales and the ability of computers to perform large scale calculations at ease, micromagnetic simulations have become a standard tool for aiding experimental studies in micromagnetism. In particular, the Object Oriented Mi- 
croMagnetic Framework, OOMMF, 11] has been cited in over 980 papers at the time of this study. For example micromagnetic simulations have been used to elucidate the domain structure of permalloy thin film circular, 12 elliptical 13

40 and rectangular 14] elements of various sizes and thicknesses. The study by Hertel highlights the region of single domain permalloy elements, noting the importance of single domain structures for technological applications. [14]

One such area that employs single domain islands is that of artificial spin ice (ASI); the islands behave as macrosized spins and allow for the effects of geomet45 rical frustration to be directly visualized by magnetic imaging techniques. [15, 16] The pioneering works in ASI examined islands of permalloy in a square pattern [16] and hexagonal networks of cobalt, [15] however the systems were static and dynamics were induced through the application of external fields [17]. Interest in a thermalised ASI system grew; it has been noted that an ideal material

50 for thermal activation would exhibit simultaneously a high magnetic moment per atom and a relatively low Curie temperature. 18] Gadolinium, possessing both of these attributes, is therefore a possible candidate for fabrication of a thermally active frustrated system.

As the properties of ferromagnetic materials, including gadolinium, are affected by the choice of shape, size, and growth conditions, [19, 20] an extensive computational analysis is here carried out to inform further experimental work on nanopatterned gadolinium. The net long axis moment of rectangular gadolinium thin film elements with varying aspect ratio and thicknesses are investigated by the use of the OOMMF[11] micromagnetic simulations package. The magnetization patterns of the islands and the effect of the shape and magnetocrystalline anisotropy involved in domain formation are analysed.

\section{Experimental}

The relaxed magnetization states of rectangular thin film gadolinium elements of varying side length, thickness, and aspect ratio were simulated using the Object Oriented MicroMagnetic Framework, OOMMF.[1] The lowest en- 
ergy configurations for given dimensions were used to determine the ground state magnetization pattern of gadolinium nanoislands, and the region of single domain islands was identified. The impact of thickness and out-of-plane anisotropy on the transition from a high remanence state to a flux closure pat70 tern was investigated.

The simulation parameters used to reflect hcp gadolinium are based on the magnetic properties found in literature. The exchange stiffness constant was determined as $A=\frac{k_{B} T_{C}}{2 a}$ where $k_{B}$ is the Boltzmann constant, $T_{C}$ is the Curie temperature of gadolinium $(293 \mathrm{~K})$ and the lattice parameter of hcpgadolinium is $a=3.64 \AA$. [9] This gives an approximate exchange stiffness constant $A=5.62 \times 10^{-12} \mathrm{Jm}^{-1}$. A saturation magnetization value of $M_{S}=2.077 \times 10^{6} \mathrm{Am}^{-1} \quad$ 9] and a uniaxial magnetocrystalline anisotropy $K_{u}=1.7 \times 10^{5} \mathrm{Jm}^{-3} \quad$ [21] acting preferentially along the c-axis 21, 22, 23] were used. A damping parameter of $\alpha=0.5$ was used; this is a relatively large value which allows the system to converge to an energy equilibrium in a more reasonable time [11] and is an appropriate value for an investigation that is not focussed on dynamics.

The dimensions of the gadolinium islands studied are defined in figure 1 , the long axis is positioned to align with the $\mathrm{x}$-axis, the short axis in the $\mathrm{y}$-direction 85 and the thickness of the island is given by the range in the z-direction. The corresponding aspect ratio of the islands is defined as the length of the island in the $\mathrm{x}$-direction divided by the width of the island in the $\mathrm{y}$-direction.

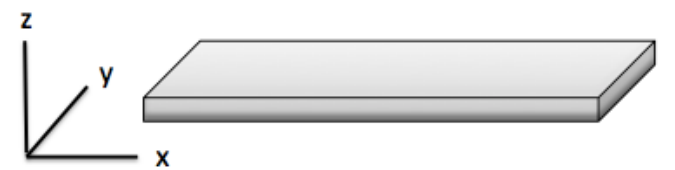

Figure 1: The orientation of a rectangular element used in the OOMMF model.

In order to best gauge the behavior of nanopatterned gadolinium, the extreme scenarios of a fully randomized grain structure (non-textured) and a grain 
structure with a common direction for the c-axis (textured) were examined. This was implemented in the simulations by setting the magnetocrystalline anisotropy to zero in the non-textured case, to model the effective cancellation of the magnetocrystalline anisotropy from a set of randomly oriented grains. The same results could be achieved by discretizing the islands and assigning the anisotropies such that they effectively cancel each other out, however this would be computationally much more expensive. In the textured case the magnetocrystalline easy axis (c-axis) was set parallel to the z-axis, reflective of the thin film behavior of the magnetocrystalline anisotropy of gadolinium reported by Scheunert et al. 9] These two treatments of the magnetocrystalline anisotropy bookends the expected behavior of fabricated structures.

In order to determine the lowest energy configuration of the magnetic structures the dimensions of the gadolinium islands were translated into the OOMMF solver; three different random starting states and a fully magnetized state were allowed to relax, using the demagnetization protocol in the software. Upon full relaxation the moment along the long axis and the energy of the associated magnetic arrangement were recorded, and the magnetization pattern of the lowest recorded energy of the four was assumed to be the ground state configuration. For smaller states, a random initial state could be resolved into a single or multi-domain energy minimum however the larger islands could find an energy equilibrium in a metastable state; this was confirmed when an initially magnetised larger island was allowed to relax and returned a lower energy state. It became apparent that a series of varying initial configurations was the best way to acquire the energy minimum.

As part of the micromagnetic solver, the input structure is discretized; the cell size is typically smaller than the exchange length of the material examined. The calculation of the exchange length has been a source for debate, 24] with calculations showing values for gadolinium between 1.44 and $5 \mathrm{~nm}$; a cell size smaller than this allows the accurate resolution of domain walls and micromagnetic features. The results presented in this paper were all acquired using the 120 cell size of $1 \mathrm{~nm}^{3}$. The majority of the simulations were also carried out with a 

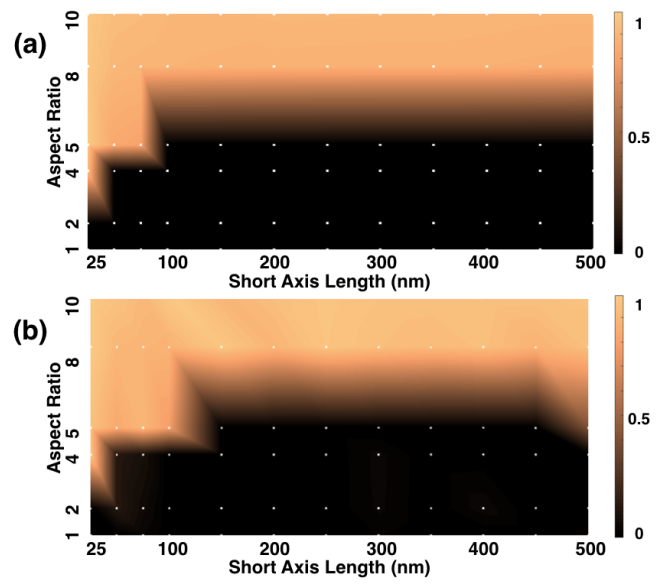

Figure 2: (Color online) Color maps showing the net moment pointing along the long axis of $15 \mathrm{~nm}$ thick (a) textured gadolinium and (b) non-textured gadolinium rectangles, in relation to short axis length and aspect ratio. White markers indicate geometries for which simulation was run, with a shading interpolation carried out in between. The simulations used the $1 \mathrm{~nm}$ discretisation. Some of the corresponding micromagnetic states are shown in figure 4

cell size of $(5 \mathrm{~nm})^{3}$, with the same qualitative results. The images provided are of the relaxed ground state; the center-slice is used unless otherwise specified.

\section{Results}

The data sets for the results shown in this paper have been made available on Mendelev data [25].

The color maps shown in figure 2 illustrate the fraction of long-axis-aligned magnetic moments in $15 \mathrm{~nm}$ thick gadolinium islands, for (a) the textured and (b) the non-textured case, according to their short axis length (x-axis) and aspect ratio (y-axis). The markers on the map illustrate the returned values of these moments, with a shading interpolation applied between these points.

The net fraction of moments acting along the long axis is used to determine whether the ground state of the system tends towards a highly remanent state or a stray-field minimizing multi-domain state. In this study, a net moment along the long axis of $0.85 M_{S}$ is taken as the threshold into 'single-domain' 


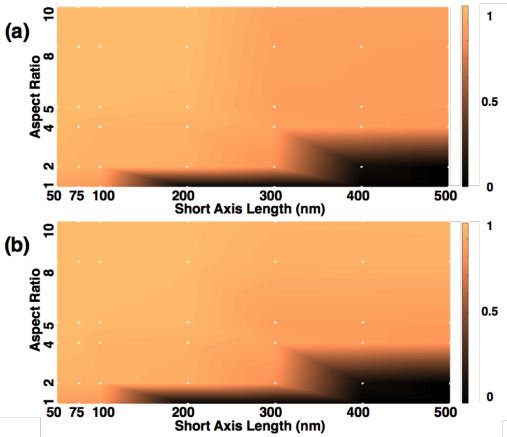

Figure 3: (Color online) Color maps showing the long axis moment of $15 \mathrm{~nm}$ thick (a) textured gadolinium and (b) non-textured gadolinium rectangles, in relation to short axis length and aspect ratio, when relaxed from a fully magnetized state; see also figure 2

behavior. The discrepancy between a fully saturated long axis moment can be attributed to edge effects; other studies have further clarified whether the patterns are C-state, S-state or flower state. 114] However, since this study is primarily concerned with the region of stability for a high magnetic moment of the nanobars, such distinctions are not made for the color maps in this paper.

As can be seen from figure 2, for both the textured and non-textured cases the single-domain state is an energetically favorable micromagnetic state at high aspect ratios (10:1) and medium aspect ratios (5:1) in combination with smaller short axis lengths (below $75 \mathrm{~nm}$ ). Square 1:1 aspect ratio gadolinium islands are observed to have an almost zero net magnetic moment along the $\mathrm{x}$-axis as they form a flux closure state, the Landau configuration, see figure 4(b). The larger rectangular islands can form multi-domain states with several flux closure patterns as illustrated in figure 4(c) and figure 6(b).

The long axis magnetization of the gadolinium islands that resulted when relaxing the system from fully magnetized states are shown as color maps in figure 3 comparison with figure 2 shows that starting from a fully magnetized state extends the stability region of large long axis moments to most dimensions investigated; the exceptions are islands with a 1:1 aspect ratio of length $200 \mathrm{~nm}$ or more and those with a 2:1 aspect ratio with short axis length greater than 
(a)

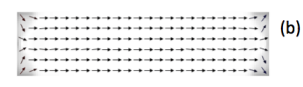

(c)
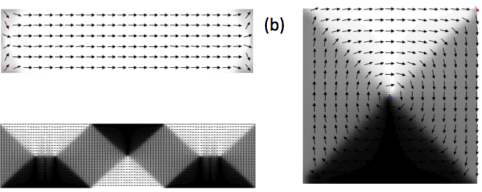

Figure 4: $\mathrm{X}-\mathrm{Y}$ view of magnetization patterns in three different $15 \mathrm{~nm}$ thick textured Gd islands from figure 2(b). The white/black coloration represent the magnitude of the moment aligned with the long axis pointing towards the right/left in the figure. (a) A highly remanent state observed in the smaller volumes at medium aspect ratios and at higher aspect ratios throughout, represented by a $300 \mathrm{~nm}$ x $75 \mathrm{~nm}$ island; (b) a Landau configuration in which the flux closure pattern minimizes stray fields outside the island, represented by a $200 \mathrm{~nm}$ x 200 $\mathrm{nm}$ island; (c) a multi-domain structure seen in the larger islands, consisting of several flux closure domain arrangements, represented by a $1200 \mathrm{~nm}$ x $300 \mathrm{~nm}$ island.

or equal to $400 \mathrm{~nm}$.

Since a short axis length of $75 \mathrm{~nm}$ marked the boundary between single and multi domain states at smaller aspect ratios and could be realistically patterned, this short axis length was used for further simulations were the thickness was varied. Figure 5 shows what fraction of the magnetic moment for (a) nontextured (b) textured gadolinium nanoislands of three different aspect ratios $(5 / 10 / 20: 1)$ is pointing in a common direction along the long axis after relaxation from an initially magnetised state, for thicknesses from $15 \mathrm{~nm}$ to $60 \mathrm{~nm}$. With increasing thickness, the fraction of the magnetic moment of the nanobar that is aligned with the long axis decreases. This is the case for all three aspect ratios explored for the chosen short axis length of $75 \mathrm{~nm}$; however, the phenomenon is most pronounced in the 5:1 aspect ratio islands. As can be seen in figure 6 the lowest energy magnetization state for this aspect ratio is a single domain for thicknesses below $30 \mathrm{~nm}$, but multi-domain states have the lowest energy at thicknesses above $30 \mathrm{~nm}$; this is the case in both the textured and non-textured simulations. The differences in magnetization patterns between textured and non-textured gadolinium nanobars become more apparent as the thicknesses of the structures increase. Accordingly, the ultra-high aspect ratio of 20:1 (75 nm $\times 1500 \mathrm{~nm})$ sees a highly remanent ground state configuration 

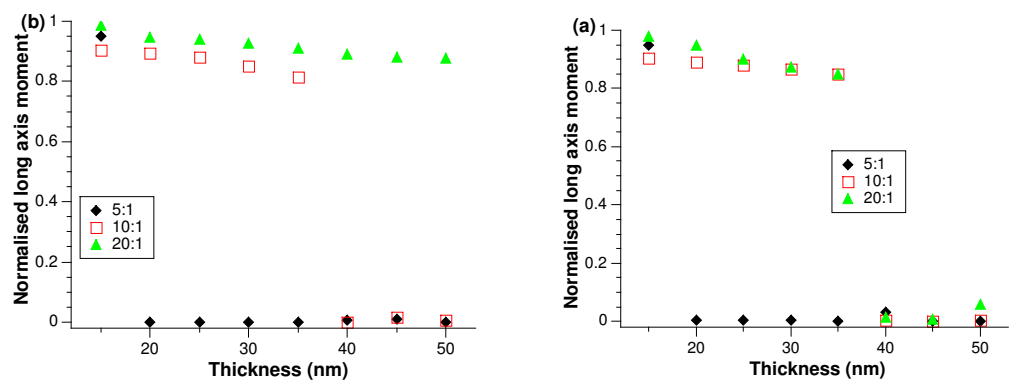

Figure 5: (Color online) The variation in net long-axis moment with increasing island thickness associated with the lowest energy configuration of (a) textured and (b) non-textured gadolinium islands of short axis length $75 \mathrm{~nm}$ and aspect ratios 5:1 (•), 10:1 ( $\square$ ), and 20:1 $(\mathbf{\Lambda})$.

in the non-textured case but a demagnetised state is assumed when examining the textured case.

The predicted micromagnetic state of a $375 \mathrm{~nm} \times 75 \mathrm{~nm} \times 55 \mathrm{~nm}$ textured nanobar is shown in figure 7. Figure 7(a) shows a y-z cross section through the island; figure 7 (b) is an x-z slice through the center of the island; and figure 7 (c) shows the central $\mathrm{x}-\mathrm{y}$ slice of the island. The combination of a magnetocrystalline anisotropy with easy axis along the z-direction and a thickness of similar magnitude to the short axis length result in a preference for the magnetic moments to align along the z-axis, forming flux closures in the z-direction. This is illustrated by the $y-z$ cross-section (figure $7(\mathrm{a})$ ) where a flux closure loop is clearly visible; the blue (dark gray) shading indicates a component of magnetization along the positive z-axis and conversely a red (light gray) pixel indicates a component of magnetization along the negative z-axis.

\section{Discussion}

From the results we can see that the dimensions of gadolinium nanobars studied show a transition from a regime wherein the shape anisotropy and exchange interaction dominates the orientation of spins, resulting in a highly remanent 


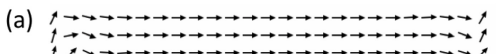

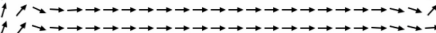

(b)

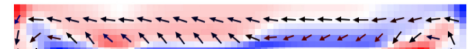

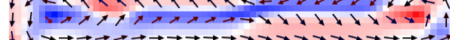

if $\rightarrow \rightarrow \rightarrow \rightarrow \rightarrow \rightarrow \rightarrow \rightarrow \rightarrow \rightarrow \rightarrow \rightarrow x \rightarrow \rightarrow \rightarrow \rightarrow \rightarrow \rightarrow \rightarrow+$

Figure 6: (Color online) Micromagnetic state in the x-y plane of two different thicknesses of a $375 \mathrm{~nm} \times 75 \mathrm{~nm}$ textured Gd nanobar, featured in figure 5(b). (a) The lowest energy configuration at a thickness of $15 \mathrm{~nm}$. White pixels represent an absence of a vertical component to the magnetization vectors, making this a highly remanent state. (b) The magnetization arrangement for a thickness of $30 \mathrm{~nm}$. Here, the colored pixels show a rotation of the magnetization vectors away from the horizontal: blue (dark gray), upwards in the out-of-plane direction; and red (light gray), downwards.

state, to a regime where minimization of the stray field is preferred and magnetization aligns parallel with outer surfaces, forming multi-domain magnetization patterns.

As already discussed, there are many factors to be considered in the formation of domains such as size, shape and composition. For gadolinium nanobars as for other ferromagnetic thin film islands, [14, 26, 27. we can see that the aspect ratio is a critical parameter, with high length to width ratios necessary to induce a highly remanent state. Alongside this, in pursuit of a single domain nanobar, the magnetic volume plays a role; for a fixed thickness of $15 \mathrm{~nm}$, structures of short axis length $75 \mathrm{~nm}$ and less were showing single domain ground states with aspect ratios of 5:1, compared with a short axis length of $100 \mathrm{~nm}$ or more that need at least 8:1 length to width ratio. The aspect ratio necessary to induce a highly remanent state in gadolinium is significantly higher than that used in thin film islands of permalloy, where a C-state, one variant of a highly remanent state, was shown to be the ground state magnetization pattern for a 2:1 $150 \mathrm{~nm}$ short axis island. 14 The high remanence state in cobalt nanostructures is also possible in aspect ratios of 2:1 at short axis lengths of $100 \mathrm{~nm}$ and above 5:1 at the larger sizes; 26] further demonstrating the different treatment gadolinium requires in obtaining single domain states. The combination of the smaller ex- 


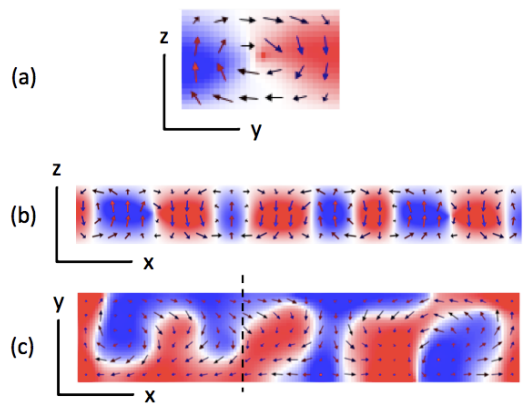

Figure 7: (Color online) A series of slices through a textured 5:1 aspect ratio 75 nm short axis Gd island of thickness $55 \mathrm{~nm}$. (a) The $\mathrm{y}-\mathrm{z}$ slice shows a flux closure pattern acting along the vertical plane and is taken along the dashed line in (c). (b) The $x-z$ slice through the center of the island presents a sideways perspective to the $\mathrm{y}-\mathrm{z}$ slice, the lack of a uniform direction to the moments showing the energetically favorable state is no longer a highly-remanent structure; the top down view of the $\mathrm{x}-\mathrm{y}$ slice (c) shows the degree of vertical alignment of the moments through the red (light gray) and blue (dark gray) pixel color. Here, the vertical flux closure patterns are again hinted at.

change interaction and larger saturation magnetization in gadolinium, relative to that of cobalt [28, 26] and permalloy, 27, 14] means that it is energetically favorable to create domain walls in comparatively smaller islands with higher aspect ratio. In these high aspect ratio structures, the shape anisotropy is the dominant factor in domain formation.

A negative correlation between increasing thickness of the gadolinium islands and net magnetic moment along the long axis was found, indicating the shape anisotropy is not as effective in thicker films. This trend was also shown to occur in permalloy. 14 In relation to gadolinium, it is suggested that the highly remanent states are most likely to be achieved at thicknesses $25 \mathrm{~nm}$ or less since this adheres to the quasi $2 \mathrm{D}$ model where $z \ll y$; islands in thicker films behave more like 3D nanostructures. The work done by Hsu et al found the nanobars created (of size $1400 \times 70 \times 50 \mathrm{~nm}^{3}$ ) possessed a much smaller long axis magnetization than the authors expected. They argued that oxidation and permeation of fcc gadolinium through their nanobars were the factors for this observation. From the results of this study, it can be shown that they used an 
In this study the magnetocrystalline anisotropy is approximated and the easy axis is presumed to act vertically out-of-the-plane of the thin film island, as found for the films grown by Scheunert et al. 222] Some studies show at non-zero temperatures that there is a rotation of the easy direction in gadolinium; 31 a 255

appropriate aspect ratio of 20:1. However, it is suggested that a structure $50 \mathrm{~nm}$ thick may result in internal spins aligning along the z-axis, forming flux closure structures in the x-z plane, as shown for the 5:1 $75 \mathrm{~nm}$ short axis nanoisland in figure 7. In these larger volumes, the moments prefer to align parallel to surfaces, as indicated in figure 4 (b) and (c), attempting to minimize the stray field outside the volume; in the textured Gd films, the magnetocrystalline anisotropy also favors orientation along the z-axis. Previous experimental work on competing anisotropies in ferromagnetic islands includes studies of the effect in ellipses fabricated from $\mathrm{Fe} / \mathrm{Co}-$ mulitilayers, 29] and rectangular Co islands, 30] where both easy axes were in the plane of the thin film. Here we have one in-plane easy axis from shape anisotropy, with the magnetocrystalline easy axis being out-of-plane. From these previous studies and the results obtained in this paper, it can be seen that more complex domain structures result from competing anisotropies, making highly remanent states less likely.

The results of this study allow a proposition of dimensions that are likely to result in the fabrication of a single domain gadolinium nanobar, taking into consideration the experimental realities of patterning such dimensions. A high aspect ratio with a lesser short-axis length and a small thickness are key to achieving a large net long axis moment; this study showed that a $15 \mathrm{~nm}$ thick island with aspect ratio of 10:1 or 20:1 in combination with a short axis length of 50-75 nm would most likely result in a highly remanent structure. Although the nanobar could be subject to the effect of volume loss through oxidation, [8] it is presumed that the minimization of fcc content achieved by Scheunert et al [9] would assist in the production of a highly remanent state.

There has been much debate on the exact magnetic properties of gadolinium due to its sensitivity to growth conditions and choice of substrate. 19, 22 study of this effect is beyond the scope of this paper. The non-textured case 
was also introduced assuming the completely random orientation of the grains in gadolinium. However, with grain sizes of the order 20-30 nm (found by Scheunert et al [9]) a discrepancy with the assumed completely random nature is probable in smaller magnetic volumes.

Domain walls cost energy to create, since they involve the rotation of neighboring spins away from the favored parallel alignment. Thus, the same volume of domains and domain walls in two different geometric arrangements can result in very similar energies; this has for example be seen in the experimental work of Gunnarsson et al 32] and in Hertel's [14 micromagnetic modelling work. Hence there is a degeneracy in the ground states of larger structures, and the simulations cannot be used to predict an exact configuration. For example, the domain structure observed in figure 4 (c) is one of several possible flux closure arrangements with similar energies. Similarly, the smaller exchange length possessed by gadolinium relative to other ferromagnets allows domain walls to form in smaller structures; hence single domain states only appear at smaller dimensions and higher aspect ratios.

The results in this study are predicated upon the assumptions made in the simulations of the ferromagnetic nanopatterned gadolinium, but they are not indicative of every gadolinium sample produced. Out-of-plane anisotropy is in 275 some ways the worst case scenario for single domain structures as it is most likely to favor an out-of-plane magnetization. The zero magnetocrystalline anisotropy simulations are a reasonable approximation of a random polycrystalline film, or a case of c-axis texture (out-of-plane) with easy plane anisotropy. These simulations therefore represent two of the most probable magnetocrystalline anisotropy configurations in ferromagnetic thin film elements of gadolinium.

\section{Conclusion}

This work examines the ground state magnetization pattern for ferromagnetic gadolinium thin film structures and shows a range of dimensions that could be used for creation of a highly remanent gadolinium thin film element. The re- 
285 anisotropy in increasingly thicker gadolinium nanoislands, with a critical thickness of $25 \mathrm{~nm}$ marking the transition from a typically highly remanent state in 5:1 aspect ratio islands to a multidomain state. This study, in conjunction with a thin film growth recipe that minimizes fcc gadolinium, [9] provides a guide achieved experimentally.

\section{References}

[1] S. Dan'kov, A. Tishin, V. Pecharsky, K. Gschneidner, Magnetic phase transitions and the magnetothermal properties of gadolinium, Physical Review B 57 (6) (1998) 3478-3490. doi:10.1103/PhysRevB.57.3478.

[2] D. Roberts, W. L. Zhu, C. M. Frommen, Z. Rosenzweig, Synthesis of gadolinium oxide magnetoliposomes for magnetic resonance imaging, Journal of Applied Physics 87 (9) (2000) 6208. doi:10.1063/1.372656.

[3] J. V. Harkins, P. E. Donovan, The structure and magnetization of gadolinium/molybdenum multilayers, Journal of Physics: Condensed Matter 8 (6) (1996) 685. doi:10.1088/0953-8984/8/6/009

[4] C. Ward, G. Scheunert, W. R. Hendren, R. Hardeman, M. A. Gubbins, R. M. Bowman, Realizing a high magnetic moment in Gd/Cr/FeCo: The role of the rare earth, Applied Physics Letters 102 (9) (2013) 092403. doi: $10.1063 / 1.4794820$

[5] J. S. Jiang, C. L. Chien, Magnetization and finite-size effects in Gd/W multilayers, Journal of Applied Physics 79 (8) (1996) 5615. doi:10.1063/ 1.362259 .

[6] R. P. Cowburn, Property variation with shape in magnetic nanoelements, 310 Journal of Physics D: Applied Physics 33 (1) (1999) R1-R16. doi:10. 1088/0022-3727/33/1/201. 
[7] F. Döbrich, J. Kohlbrecher, M. Sharp, H. Eckerlebe, R. Birringer, A. Michels, Neutron scattering study of the magnetic microstructure of nanocrystalline gadolinium, Physical Review B - Condensed Matter and Materials Physics 85 (9) (2012) 094411. doi:10.1103/PhysRevB.85. 094411

[8] C. J. Hsu, S. V. Prikhodko, C. Y. Wang, L. J. Chen, G. P. Carman, Magnetic anisotropy in nanostructured gadolinium, Journal of Applied Physics 111 (5) (2012) 053916. doi:10.1063/1.3691220.

${ }_{320}$ [9] G. Scheunert, W. R. Hendren, C. Ward, R. M. Bowman, Magnetization of 2.6 T in gadolinium thin films, Applied Physics Letters 101 (14) (2012) 142407. arXiv:1209.5617, doi:10.1063/1.4757126.

[10] M. Chakravorty, A. K. Raychaudhuri, Low field magnetoresistance of gadolinium nanowire, Journal of Applied Physics 115 (5) (2014) 2012-2017. doi:10.1063/1.4864124.

[11] M. Donahue, D. Porter, OOMMF User's Guide, Version 1.0 Interagency Report NISTIR 6376, Tech. rep. (2002). URL http://math.nist.gov/oommf /

[12] H. Jonathan Kin, E. al., Concentric domains in patterned thin films with perpendicular magnetic anisotropy, EPL (Europhysics Letters) 64 (6) (2003) 810. doi:10.1209/epl/i2003-00630-6.

[13] P. Warnicke, S. Felton, K. Gunnarsson, P. Svedlindh, Simulations of magnetic microstructure in thin film elements used for programmable motion of magnetic particles, Journal of Magnetism and Magnetic Materials 303 (2 SPEC. ISS.) (2006) 294-298. doi:10.1016/j.jmmm.2006.01.085.

[14] R. Hertel, Thickness dependence of magnetization structures in thin Permalloy rectangles, Zeitschrift für Metallkunde 93 (10) (2002) 957-962. doi:10.3139/146.020957. 
[15] M. Tanaka, E. Saitoh, H. Miyajima, T. Yamaoka, Y. Iye, Magnetic inter-

[20] A. G. Kolhatkar, A. C. Jamison, D. Litvinov, R. C. Willson, T. R Lee, Tuning the magnetic properties of nanoparticles, International Jour-

I

[21] S. Abdelouahed, M. Alouani, Magnetic anisotropy in Gd, GdN, and GdFe2 tuned by the energy of gadolinium 4f states, Physical Review B 79 (5) (2009) 054406. doi:10.1103/PhysRevB.79.054406

[22] G. Scheunert, C. Ward, W. R. Hendren, a. a. Lapicki, R. Hardeman, talline ordering on magnetic properties of high moment rare earth metals 
and alloys, Journal of Physics D: Applied Physics 47 (41) (2014) 415005. doi:10.1088/0022-3727/47/41/415005.

[23] G. L. F. Fraga, P. Pureur, L. P. Cardoso, Impedance and initial magnetic permeability of gadolinium, Journal of Applied Physics 107 (5) (2010) 053909. doi:10.1063/1.3288696.

[24] J. M. Coey, Magnetism and magnetic materials, Cambridge University Press, 2010.

[25] M. McMullan, S. Felton, Gadolinium micromagnetic simulations, v2, http: //dx.doi.org/10.17632/r5bdsysd6m.2, doi:10.17632/r5bdsysd6m.2.

[26] E. Seynaeve, G. Rens, A. V. Volodin, K. Temst, C. Van Haesendonck, Y. Bruynseraede, Transition from a single-domain to a multidomain state in mesoscopic ferromagnetic Co structures, Journal of Applied Physics 89 (1) (2001) 531-534. doi:10.1063/1.1324687.

[27] R. D. Gomez, T. V. Luu, A. O. Pak, K. J. Kirk, J. N. Chapman, Domain configurations of nanostructured Permalloy elements, Journal of Applied Physics 85 (8) (1999) 6163. doi:10.1063/1.370030.

[28] D. Grujicic, B. Pesic, Micromagnetic studies of iron microbars prepared by nanoimprint lithography and electrodeposition, Thin Solid Films 485 (1-2) (2005) 218-223. doi:10.1016/j.tsf.2005.03.042.

[29] S. Felton, K. Gunnarsson, P. Svedlindh, M. Hanson, O. Kazakova, Micromagnetic studies of $\mathrm{Fe} / \mathrm{Co}$ ellipses with competing anisotropy contributions, Journal of Magnetism and Magnetic Materials 272-276 (SUPPL. 1) (2004) 2003-2004. doi:10.1016/j.jmmm.2003.12.428.

[30] T. Kohda, Y. Otani, V. Novosad, K. Fukamichi, S. Yuasa, M. Nyvlt, T. Katayama, Shape effect on the magnetic anisotropy of an array of epitaxial (10-0) Co dots, IEEE Transactions on Magnetics 35 (5 PART 2) (1999) 3472-3474. doi:10.1109/20.800561. 
[31] H. E. Nigh, S. Legvold, F. H. Spedding, Magnetization and Electrical Resistivity of Gadolinium Single Crystals, Phys. Rev. 132 (3) (1963) 1092-1097. doi:10.1103/PhysRev. 132.1092.

[32] K. Gunnarsson, P. E. Roy, S. Felton, J. Pihl, P. Svedlindh, S. Berner, H. Lidbaum, S. Oscarsson, Programmable motion and separation of single magnetic particles on patterned magnetic surfaces, Advanced Materials 17 (14) (2005) 1730-1734. doi:10.1002/adma.200401880. 